\title{
Article \\ A Variant of the Necessary Condition for the Absolute Continuity of Symmetric Multivariate Mixture
}

\author{
Evgeniy Anatolievich Savinov ${ }^{+}($D
}

check for

updates

Citation: Savinov, E. A Variant of the Necessary Condition for the Absolute Continuity of Symmetric Multivariate Mixture. Mathematics 2021, 9, 1505. https://doi.org/10.3390/math9131505

Academic Editor: Mark Kelbert

Received: 30 April 2021

Accepted: 25 June 2021

Published: 27 June 2021

Publisher's Note: MDPI stays neutral with regard to jurisdictional claims in published maps and institutional affiliations.

Copyright: (c) 2021 by the authors. Licensee MDPI, Basel, Switzerland. This article is an open access article distributed under the terms and conditions of the Creative Commons Attribution (CC BY) license (https:/ / creativecommons.org/licenses/by/ $4.0 /)$.
Financial University under the Government of the Russian Federation, 125993 Moscow, Russia; easavinov@fa.ru + Current address: 49 Leningradsky Prospekt, GSP-3.

Abstract: Sufficient conditions are given under which the absolute continuity of the joint distribution of conditionally independent random variables can be violated. It is shown that in the case of a dimension $n>1$ this occurs for a sufficiently large number of discontinuity points of one-dimensional conditional distributions.

Keywords: a multidimensional mixture; de Finetti's theorem; permutation values; reliability theory; singular copulas

\section{Introduction}

In this note, we study multidimensional mixtures of the form

$$
F_{n}\left(x_{1}, \ldots, x_{n}\right)=\int_{-\infty}^{\infty} \prod_{k=1}^{n} F\left(x_{k} \mid r\right) \mu(d r), \quad x_{k} \in[0,1],
$$

which coincide, due to de Finetti's theorem, with the class of joint distributions of symmetrically dependent (or infinitely permutable) random variables. Such models arise, in particular, in the mathematical theory of financial risks and in the theory of reliability, where they describe the behavior of systems consisting of identical components under the influence of random external conditions.

There are many works devoted to the study of conditions under which conditional marginal distributions, or vice versa, the original symmetric law, belong to one or another class of distributions or type. For example, in the works [1,2], the type of dependence between conditionally independent random variables with a distribution function (1) is studied with additional requirements for conditional distribution functions.

In particular, it is mentioned that a sufficient condition for the absolute continuity of the mixture is the absolute continuity of the conditional distribution functions. A similar statement is also found in [3-5] for more general mixtures.

As the following simple example shows, this condition is not necessary. In the case of $n=1$, even continuity is not required. It suffices to take as $\mu$ the Lebesgue measure on $[0,1], F(x \mid r):=\mathbf{1}_{[r, 1]}(x)$. Then $F_{1}(x)$ is the uniform distribution function of $U[0,1]$.

This note deals with a variant of the necessary condition for the absolute continuity of the mixture (1), which has not been presented in the literature before. More specifically, if too many univariate conditional distribution functions in the family have discontinuity points, then mixture (1) will not be absolutely continuous.

The result is important for statistical applications to the theory of reliability and finance. The formulation of the theorem is motivated by the following considerations.

Let us consider the system of random variables $X_{1}, \ldots, X_{n}$ as life lengths of $n$ identical components of a complex system operating in a random environment. Let them be i.i.d. for a given state of the environment. Such a system is considered, for example, in [6]. Suppose additionally that at some random moment in time, depending on the state of 
the environment, each component of the system is independently affected (or in many cases may be affected) by some destructive effect leading to component failure with probability $p \in(0,1)$. The question arises, in particular, is it possible to use kernel density estimation as a nonparametric estimate of the joint distribution of their life lengths based on observations of the components of such a system? Will it be possible to apply the Rosenblatt transformation (see [7]) to check the goodness-of-fit of the sample with such a distribution? The following theorem answers these questions in the negative.

\section{Results}

Theorem 1. Let

$$
F_{n}\left(x_{1}, \ldots, x_{n}\right)=\int_{-\infty}^{\infty} \prod_{k=1}^{n} F\left(x_{k} \mid r\right) \mu(d r), \quad x_{k} \in[0,1]
$$

the distribution function concentrated on the unit cube, and $F(x \mid r)$ is the one-dimensional distribution function on $[0,1]$ for $\mu$-almost all $r$. Let $G$ be the set of points $r$, for which the function $F_{r}(x)=F(x \mid r)$ has a discontinuity.

If $G$ is $\mu$-measurable, $\mu(G)>0$, and there is some $\mu$-measurable function $r \mapsto x(r)$, where $x(r)$ any of the discontinuity points of the function $F_{r}(x)$, then $F_{n}$ is not absolutely continuous in the case $n>1$.

Proof of Theorem 1. Define the jump function

$$
g(r)=F(x(r) \mid r)-F(x(r)-0 \mid r)>0, \quad \forall r \in G
$$

For an arbitrary arbitrarily small number $\delta>0$, choose a natural $N>\delta^{-\frac{1}{n-1}}$ and set $v:=1 / N$. Let it denote the following (measurable due to the measurability of $x(r)$ and $G$ ) subsets of the real line

$$
\begin{gathered}
R_{0, v}:=\left\{r \in G: x(r)=x_{0}\right\}, \quad R_{i, v}:=\left\{r \in G: x(r) \in\left(x_{i}-v, x_{i}\right]\right\}, \quad i=1, \ldots, N, \\
x_{i}=i v, \quad i=0,1,2, \ldots, N
\end{gathered}
$$

Obviously, $\bigcup_{i=0}^{N} R_{i, v}=G$.

Note that summing the $F$-volumes of the cubes $\left(x_{i}-v, x_{i}\right]^{n}$, we obtain

$$
\begin{gathered}
\sum_{i=0}^{N} V_{F}\left(\left(x_{i}-v, x_{i}\right]^{n}\right)=\sum_{i=0}^{N} \int_{-\infty}^{\infty}\left[F\left(x_{i}, \mid r\right)-F\left(x_{i}-v, \mid r\right)\right]^{n} \mu(d r) \\
\geq \sum_{i=0}^{N} \int_{R_{i, v}}\left[F\left(x_{i}, \mid r\right)-F\left(x_{i}-v, \mid r\right)\right]^{n} \mu(d r) \geq \sum_{i=0}^{N} \int_{R_{i, v}} g^{n}(r) \mu(d r)=\int_{G} g^{n}(r) \mu(d r) .
\end{gathered}
$$

The last integral is strictly positive since $g(r)>0$ on $G$, and $\mu(G)>0$.

Thus, we have a number $\varepsilon=\int_{G} g^{n}(r) \mu(d r)>0$ such that for any $\delta>0$ a set of measurable sets is indicated $\left\{\{0\}^{n},\left(x_{i}-v, x_{i}\right]^{n}, \quad i=1,2, \ldots, N\right\}$ with the total Lebesgue measure $N v^{n}+0=N^{-(n-1)}<\delta$, with the total $F$-volume

$$
V_{F}\left(\{0\}^{n}\right)+\sum_{i=1}^{N} V_{F}\left(\left(x_{i}-v, x_{i}\right]^{n}\right)=\sum_{i=0}^{N} V_{F}\left(\left(x_{i}-v, x_{i}\right]^{n}\right) \geq \varepsilon,
$$

which denies the absolute continuity of the function $F_{n}$. 


\section{Discussion}

In addition to the application to the theory of reliability, the result can be used, for example, to construct symmetric singular copulas along with the geometric method (see [8]). For example, for $n=2$, denoting the Lebesgue measure on [0,1] by $\mu, F(x \mid r):=$ $\mathbf{1}[r, 1](x)$ (here $x(r)=r, G=[0,1])$, we obtain $F_{2}\left(x_{1}, x_{2}\right)=\min \left\{x_{1}, x_{2}\right\}$ is a singular copula, an upper Frechet-Hoeffding bound.

Great thanks to the reviewers for their helpful comments.

Funding: This research received no external funding.

Institutional Review Board Statement: Not applicable.

Informed Consent Statement: Not applicable.

Conflicts of Interest: The author declares no conflict of interest.

\section{References}

1. Khaledi, B.E.; Kochar, S. Dependence properties of multivariate mixture distributions and their applications. Ann. Inst. Statist. Math. 2001, 53, 620-630. [CrossRef]

2. Marshall, A.W.; Olkin, I. Multivariate distributions generated from mixtures of convolution and product families. In Topics in Statistical Dependence; Institute of Mathematical Statistics: Hayward, CA, USA, 1990; pp. 371-393.

3. Belzunce, F.; Mercader, J.-A.; Ruiz, J.-M.; Spizzichino, F. Stochastic comparisons of multivariate mixture models. J. Multivar. Anal. 2009, 100, 1657-1669. [CrossRef]

4. Shaked, M.; Spizzichino, F. Positive dependence properties of conditionally independent random lifetimes. Math. Oper. Res. 1998, 23, 944-959. [CrossRef]

5. Hu, T.; Chen, J.; Xie, C. Regression dependence in latent variable models. Probab. Eng. Inf. Sci. 2006, 20, 363-379. [CrossRef]

6. Shaked, M. A concept of positive dependence for exchangeable random variables. Ann. Stat. 1977, 5, 505-515. [CrossRef]

7. Rosenblatt, M. Remarks on multivariate transformation. Ann. Math. Stat. 1952, 23, 470-472. [CrossRef]

8. Nelsen, R. An Introduction to Copulas, 2nd ed.; Springer: New York, NY, USA, 2006; 276p. 